\title{
Aprendizaje implícito en usuarios intensivos de videojuegos
}

\author{
Lucio Rehbein Felmer \\ Paula Alonqueo Boudon \\ Michael Filsecker \\ Universidad de La Frontera, Temuco, Chile
}

Resumen: El presente estudio buscó relacionar la evidencia acumulada sobre aprendizaje implícito y el potencial impacto de los videojuegos como vehiculo de tal aprendizaje. Específicamente, se buscó averiguar si la experiencia intensa y prolongada con videojuegos generaba ventajas por sobre la ausencia de tal experiencia, en situaciones donde existían las condiciones para aprender de manera implícita. El estudio comparó el desempeño de jugadores intensivos $(n=18)$, jugadores moderados $(n=15)$, y no-jugadores $(n=15)$, en dos tareas experimentales especialmente diseñadas para posibilitar el aprendizaje implícito: Una tarea de Tiempo de Reacción Serial (TRS); y una tarea de Inducción de Respuestas Direccionales (IRD). Los resultados con ambas tareas, aunque poco robustos, muestran que los jugadores intensivos, y también los moderados, presentan facilitación para captar las regularidades implícitas, o subliminalmente presentes, en estas tareas. Estos resultados se discuten en relación a la literatura sobre aprendizaje implícito y al potencial de los videojuegos como vehiculo de aprendizaje.

Palabras clave: Aptitud para aprendizaje. Juegos. Educación. Procesos cognitivos.

\section{Implicit learning in intensive videogame players}

\begin{abstract}
This study aimed to relate the available evidence on implicit learning and the potential role of videogames as a vehicle for such learning. Specifically, it was intended to inquire whether having an intense and long experience with videogames would grant some advantage over the absence of such experience, in situations where appropriate conditions for implicit learning were presented. Hence, the study compared the performance of intensive players $(n=18)$, moderate players $(n=15)$ and non-players $(n=15)$, on two specially designed implicit learning tasks: The Serial Reaction Time (SRT) task, and the Directional Response Induction (DRI) task. Even though the results from both tasks are not robust, they showed that intensive, as well as moderate players, were favored for capturing the implicit (subliminal) regularities present in these tasks. These results are discussed in terms of recent developments in the implicit learning literature, as well as in terms of videogames potential to become learning tools.
\end{abstract}

Keywords: Learnig ability. Games. Education. Cognitive processes.

\section{Aprendizado implícito em usuários intensivos de videogames}

Resumo: O estudo em questão procurou relacionar a evidência acumulada sobre o aprendizado implícito e o potencial impacto que o videogame pode produzir como veículo de tal aprendizado. De forma mais específica, procurou-se averiguar se a experiência intensa e prolongada com videogames gerava vantagens sobre a ausência da mesma, em situações nas quais existiam as condições para aprender de forma implícita. O estudo comparou o desempenho de jogadores intensivos $(n=18)$, jogadores moderados $(n=15)$, e não-jogadores $(n=15)$, em dois exercícios experimentais especialmente desenhados para possibilitar o aprendizado implícito: uma tarefa de Tempo de Reação Serial (TRS) e uma tarefa de Indução de Respostas Direcionadas (IRD). Os resultados de ambos exercícios, embora pouco robustos, mostram que os jogadores intensivos, assim como os moderados, apresentam facilidades para captar as regularidades implícitas, ou presentes de forma subliminar, nestes exercícios. Estes resultados são discutidos em relação à literatura disponível sobre o aprendizado implícito e o potencial do videogame como veículo de aprendizado.

Palavras-chave: Aptidão para aprendizagem. Jogos. Educação. Processos cognitivos. 


\section{Introducción}

El aprendizaje implícito ha sido caracterizado por diversos autores como un proceso a través del cual alguna información relevante de una tarea resulta incorporada automáticamente en el desempeño del sujeto, sin que éste tenga una noción consciente de lo aprendido (Reber, 1967, 1993; Jackson \& Jackson, 1995; Cleeremans, 1997). El propio Reber, quien fuera el primer investigador en describir este fenómeno, lo caracterizó como “... la adquisición de conocimiento que tiene lugar, en ausencia de conciencia por parte del sujeto, tanto del proceso de adquisición como de la base de conocimientos finalmente adquirida." (Reber, 1993, p.12).

Por su parte, Jiménez y Méndez (1999) han enriquecido la noción original señalando que el aprendizaje implícito no sólo se manifiesta a través de un proceso de adquisición automático, sino que se manifiesta también mediante sus efectos sobre la conducta, que dependen de una recuperación inconsciente y no intencional del conocimiento relevante (Jiménez \& Méndez, 1999).

Se ha demostrado que una buena técnica experimental para comprobar la presencia de aprendizaje implícito es mediante el aprendizaje probabilístico de secuencias (Nissen \& Bullemer, 1987; Cleeremans \& McClelland, 1991; Cleeremans, 1997; Jiménez \& Méndez, 2001). En éste procedimiento experimental, se les presenta a los participantes una tarea de tiempo de reacción serial (TRS) en la cuál se les instruye para responder tan rápida y exactamente como les sea posible ante la aparición de un estímulo en una de diferentes localizaciones posibles en la pantalla de un computador. La localización de cada estímulo en cada ensayo sigue una secuencia azarosa y diferente; sin embargo, sin que los participantes lo sepan, cada cierto número de secuencias se repite una y la misma serie, estructurada como una secuencia fija de localizaciones (e.g, Cohen, Ivry \& Keele, 1990; Nissen \& Bullemer, 1987). Las investigaciones con ésta técnica han demostrado que el aprendizaje de la secuencia repetida (reflejado en tiempos de reacción significativamente menores que en el caso de las secuencias azarosas) es, en su mayoría inconsciente, y que se produce de manera independiente de las intenciones de los participantes por aprender (Jiménez, Vaquero \& Lupiáñez, 2006).

En el contexto de la existencia comprobada de un aprendizaje incidental, no intencionado, capaz de captar las regularidades del ambiente y la experiencia sin el control consciente del sujeto (Barlow, 2001; Shepard, 1994), como es el aprendizaje implícito recién descrito, en el presente estudio nos preguntamos hasta qué punto un aprendizaje de este tipo puede tener lugar en un ámbito emergente de actividades recreativas como son los videojuegos (Alonqueo \& Rehbein, no prelo). Los videojuegos constituyen un fenómeno lúdico relativamente reciente que forma parte importante de la cultura infantil y juvenil a nivel mundial (Estalló, 1997, 2000; Gee, 2003). Los estudios referidos no sólo dan cuenta de la alta frecuencia de uso de los videojuegos (en adelante VJ), sino que también indican que, a causa de su carácter lúdico, constituyen una experiencia altamente atractiva y motivadora para los usuarios, al punto que una gran proporción de adolescentes y jóvenes pasan una fracción importante de su jornada semanal jugando (Alonqueo \& Rehbein, no prelo; Diez y cols, 2004).

Por otro lado, se plantea que el uso de VJ potencia el desarrollo de una serie de habilidades entre las que se destacan las de tipo espacial (Okagaki \& Frensch, 1994), destrezas manipulativas y agilidad de respuesta, estrategias de resolución de problemas (Alfageme \& Sánchez, 2003). Además, este tipo de juegos estimula el desarrollo de la atención, razonamiento inductivo, habilidades psicomotrices, búsqueda de información, habilidades organizativas para resolver tareas simultáneas y, en los usuarios más avanzados, habilidades metacognitivas (Gros y cols, 1998). Por otra parte, los propios usuarios perciben que esta práctica fomenta el desarrollo de reflejos (64\%), agilidad mental (52\%), y atención e imaginación (40\%), (Diez y cols, 2004; Alonqueo \& Rehbein, no prelo).

La presente investigación tuvo por objetivo poner a prueba la hipótesis de que el uso intensivo de videojuegos constituye una fuente de aprendizajes implícitos. Este objetivo se buscó alcanzar a través 
de la administración de dos tareas de aprendizaje implícito, una de tiempo de reacción serial (TRS) y la otra de inducción subliminal (prime) de respuestas direccionales (IRD) a tres grupos de adolescentes, con diferentes niveles de experiencia como videojugadores (intensivos, moderados y no jugadores), en un diseño cuasi-experimental con participantes seleccionados a partir del estudio de hábitos y preferencias de uso de videojuegos de Alonqueo y Rehbein, no prelo

\section{Método}

\section{Experimento 1: Tiempo de Reacción Serial}

En el primer experimento se utilizó un paradigma de tiempo de reacción serial (TRS) para comparar el desempeño de estudiantes pertenecientes a tres grupos diferentes en cuanto a su experiencia con videojuegos. Se esperaba que los estudiantes en el grupo de videojugadores intensivos presentaran tiempos de reacción significativamente menores que los estudiantes en los otros dos grupos frente a series de estímulos inadvertidamente repetidas, presentadas de manera intercalada con otras series de estímulos, generadas con secuencias azarosas de los mismos estímulos y presentada con menos frecuencia. En cambio, no se esperaban diferencias estadísticamente significativas en los tiempos de reacción de las respuestas de los otros dos grupos al comparar su desempeño entre las series frecuentes y esporádicas. El supuesto a la base de este experimento fue que quienes tuvieran una intensa y prolongada experiencia en reaccionar rápida y certeramente frente a las diversas demandas de respuesta inherentes a la ejecución de diferentes juegos electrónicos, tendrían ventaja por sobre los jugadores menos experimentados y los no jugadores para captar regularidades en la secuencia de los estímulos, incorporadas en el procedimiento experimental.

\section{Participantes}

Los participantes fueron seleccionados a partir de un colectivo de 864 estudiantes (430 mujeres y 434 hombres) de primero y segundo año de enseñanza secundaria, matriculados establecimientos educativos particulares o particular-subvencionados de la ciudad de Temuco, que fueron encuestados con el propósito de conocer sus hábitos y preferencias en relación al uso de videojuegos (Alonqueo \& Rehbein, no prelo).

En términos específicos tomaron parte en este estudio tres grupos de estudiantes. El primer grupo, integrado por estudiantes que informaron jugar $20 \mathrm{o}$ más horas a la semana, durante el último año (Jugadores intensivos, $n=18$ ). El segundo grupo, quedó integrado por estudiantes que jugaban en promedio no más de 3 horas semanales, durante el último año (Jugadores moderados, $n=15$ ). Y el tercer grupo, conformado por estudiantes, usuarios habituales de computador, pero con esporádica o nula experiencia con videojuegos (No-jugadores, $n=15$ ).

Todos los estudiantes consintieron de manera voluntaria a participar en el estudio y aceptaron acudir a la Universidad para hacer efectiva su participación.

\section{Tarea de Tiempo de Reacción Serial}

El diseño y administración de las series de estímulos así como el registro de los tiempos de reacción de cada respuesta de los participantes, se realizó mediante el uso de la aplicación computacional especializada denominada E-Prime® de Psychology Software Tools Inc. En las series de estímulos, cada ensayo consistía en la presentación de uno de los símbolos “?”, “\#”, “@” o “i” en el centro de la pantalla, y el participante debía responder con la tecla asignada a cada estímulo lo más rápido que le fuera posible. Las teclas de respuesta fueron A, S, L y $\tilde{\mathrm{N}}$, asignadas a los símbolos “?”, “\#”, “@” o “i”, respectivamente. Sobre cada tecla se pegó un adhesivo que mostraba el símbolo asignado a esa tecla. Existían dos tipos de series, las frecuentes (FREC) y las infrecuentes (INFREC), que se alternaban en una proporción 8:2 al interior de cada bloque. La serie FREC contenía la secuencia de estímulos correspondiente a 123421413243 , donde los números del 1 al 4 se corresponden con los símbolos “?”, “@”, “\#” y “i”, respectivamente. A su vez, la serie INFREC consistía en la secuencia 423143213412.

Entre un bloque y otro había un breve descanso, intervalo que el propio participante terminaba apretando la barra espaciadora cuando se 
sentía preparado para continuar. Una vez finalizado el tercer bloque, se instruía al/a la participante que respondiera a las tres series siguientes y que cuando no aparecieran los caracteres en pantalla, continuara respondiendo según le pareciera correcto. La última de estas series correspondía a la serie frecuente; nada más que esta vez, sólo los tres primeros caracteres, fueron mostrados; para los restantes, aparecía la pantalla en blanco. Esta condición final buscaba medir de manera directa el aprendizaje de la serie frecuente.

\section{Procedimiento}

La administración de la aplicación con las series tuvo lugar de manera individual en una oficina especialmente habilitada en la Universidad, donde cada participante debió concurrir en una fecha y hora señalada. La administración propiamente tal tuvo una duración aproximada de 35 minutos. Al finalizar la sesión los participantes recibían un compensación monetaria equivalente al costo de la movilización y una merienda en la universidad.

\section{Resultados}

El desempeño de los tres grupos de participantes en la tarea de Tiempo de Reacción Serial fue comparado tanto en relación al número de aciertos (cantidad de veces que el sujeto apretó la tecla correcta ante la presentación de cada uno de los caracteres en la pantalla), como en relación a los tiempos de respuesta frente a la presentación de cada estímulo

Tabla 1

Medias y desviaciones estándar de respuestas acertadas de los participantes en los grupos de jugadores intensivos, jugadores moderados y nojugadores, en la tarea de Tiempo de Reacción Serial

\begin{tabular}{lrrc}
\hline \multicolumn{1}{c}{ Grupo } & $\boldsymbol{n}$ & Media & $\begin{array}{c}\text { Desviación } \\
\text { Estándar }\end{array}$ \\
\hline Intensivo & 18 & 90 & 2,9 \\
Moderado & 15 & 86 & 3,4 \\
No-jugador & 15 & 88 & 3,2 \\
\hline
\end{tabular}

Los promedios y desviaciones típicas de respuestas correctas emitidas por los participantes en la tarea de TRS se presentan en la Tabla 1.

Como es posible observar en la Tabla 1, la tasa de aciertos de los tres grupos en esta tarea fue muy similar, con medias fluctuando entre 86 y 90, y desviaciones estándar que fluctuaron entre 2,9 y 3,4, razón por la cual se omitió el cálculo de índices estadísticos.

Sin embargo, la variable dependiente considerada crítica para la detección de eventuales diferencias en el desempeño de los grupos en esta tarea fue el tiempo de reacción de los participantes de cada grupo ante los estímulos de la serie frecuente y la serie esporádica. Los promedios y desviaciones típicas de los tiempos de reacción registrados por los participantes en cada uno de los grupos del estudio se presentan en el gráfico de la Figura 1.

Con estos datos se procedió a comparar los grupos utilizando un análisis de varianza de una vía. El resultado de este análisis muestra que los grupos difieren de manera significativa significativamente menores que los del grupo de jugadores moderados, $p<0,05$. No hubo diferencias significativas entre el grupo de jugadores intensivos y el grupo de nojugadores.

Por último, los datos obtenidos en la sección final de esta tarea, donde se buscaba evaluar el recuerdo espontáneo de la serie frecuente por los sujetos en los tres grupos, los resultados fueron caóticos y no confiables debido a que la gran mayoría de los participantes se abstuvo de responder cuando dejaron de aparecer los caracteres de la serie en la pantalla.

Los resultados obtenidos con la administración de a tarea de TRS a los tres grupos de participantes que diferían en su experiencia con videojuegos confirman lo hipotetizado, en el sentido que quienes presentan una trayectoria de uso intensivo de videojuegos tendrían un desempeño con tiempos de reacción significativamente más breves con la serie frecuente que con la serie esporádica, y que ese desempeño sería significativamente diferente al de los participantes de los otros dos grupos en estudio; 


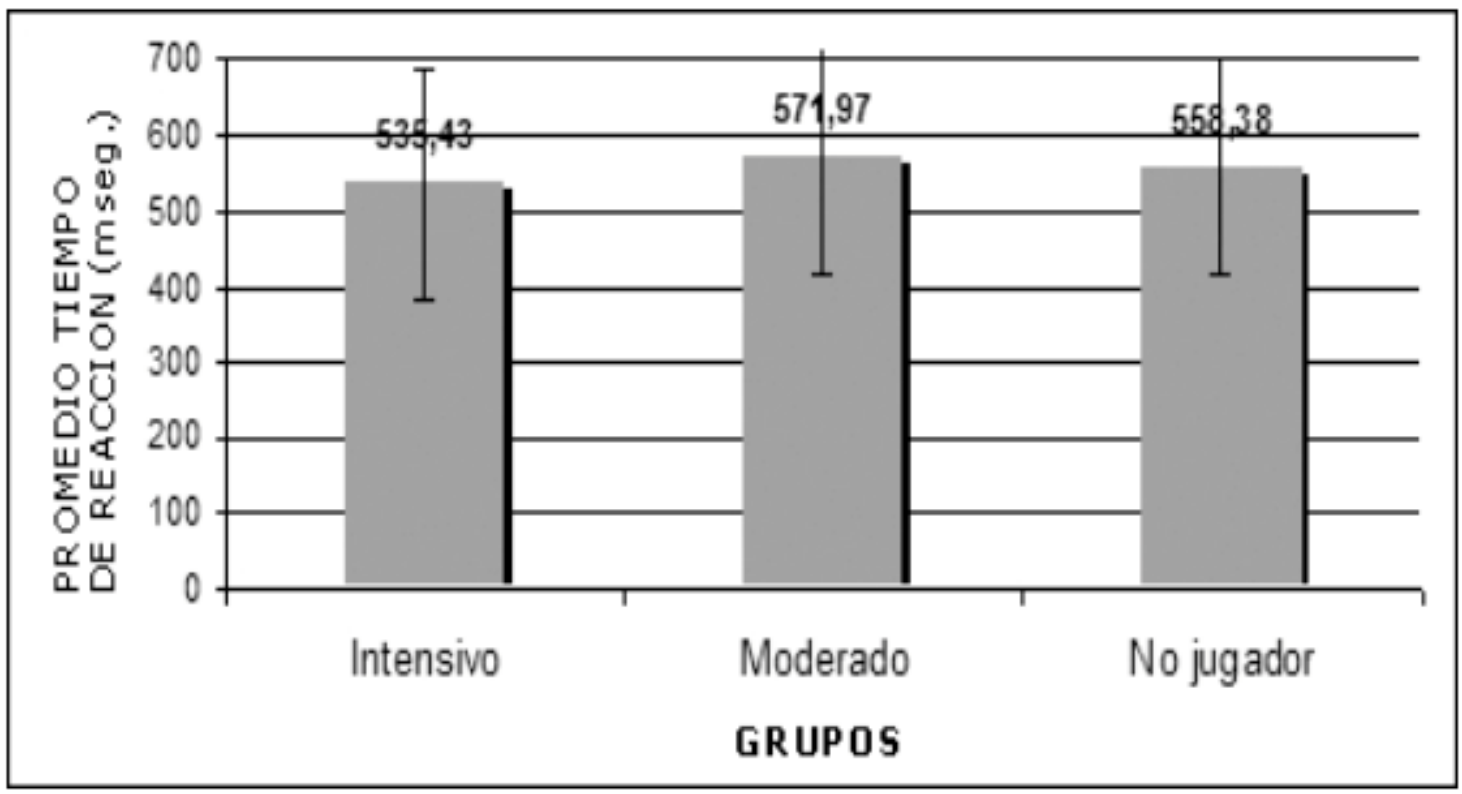

Figura 1. Promedios y desviaciones estándar de los tiempos de reacción de las respuestas en los grupos de jugadores intensivos, moderados y no-jugadores con la serie frecuente de la tarea de Tiempo de Reacción Serial.

sin embargo, sólo se encontraron diferencias significativas entre los jugadores intensivos y los moderados. Estos resultados serán discutidos más adelante en la sección correspondiente.

\section{Experimento 2: Inducción subliminal de respuestas direccionales}

Dado que en el primer experimento se comprobó la ocurrencia de aprendizaje implícito, a través de la leve reducción observada en los tiempos de respuesta de los jugadores intensivos en contraste con los jugadores moderados y no-jugadores, se buscó un procedimiento alternativo que pudiera entregar resultados más robustos e inequívocos. En términos específicos, en un segundo experimento se utilizó un estímulo inductor subliminal (prime) en una tarea donde se registraba el tiempo de reacción ante el requerimiento de señalar el punto cardinal (arriba, abajo, izquierda o derecha) en que aparecía un estimulo visual con una duración sobre umbral de 500 $\mathrm{ms}$. El supuesto en esta tarea era que los participantes con trayectoria en el uso intensivo de videojuegos tendrían ventaja sobre los participantes de los otros dos grupos en el aprovechamiento de la clave implícita o subliminal presentada, y así lograrían un desempeño más eficiente (tiempos de reacción más cortos) al momento de señalar la localización del estimulo diana (blanco o target).

\section{Participantes}

Los participantes fueron los mismos adolescentes que tomaron parte en el experimento anterior con la tarea TRS; es decir, un grupo de Jugadores intensivos, $(n=18)$; un grupo de Jugadores moderados $(n=15)$; y un grupo de No-jugadores $(n=15)$.

Tarea de Inducción subliminal (prime) de localización espacial

El diseño y administración de los estímulos presentados en esta prueba, así como el registro de los tiempos de reacción de cada respuesta de los participantes, se realizó mediante el uso de la aplicación computacional especializada denominada E-Prime ${ }^{\circledR}$ de Psychology Software Tools Inc.

La sesión experimental completa estuvo constituida por un solo bloque de 24 ensayos. Cada ensayo estaba constituido por la presentación sucesiva 
de tres estímulos consecutivos: (1) una máscara (1000 ms) consistente en un cuadrado gris de $1 \mathrm{~cm}$ cuadrado que aparecía al centro de la pantalla, (2) un prime ( $29 \mathrm{~ms}$ ) consistente en una flecha que aparecía al centro de la pantalla y (3) el target (500 ms) consistente en un cuadrado negro de $1,5 \mathrm{~cm}$ por lado en una de las 4 bordes cardinales de la pantalla de un monitor de 17 pulgadas. En la mitad de los ensayos (12) la orientación de la flecha (prime) y la ubicación del cuadrado negro (target) coincidían, y en la otra mitad no coincidían. La sucesión de los ensayos se generó de manera aleatoria.

Durante la sesión experimental, cada participante debía permanecer con la vista puesta en un punto de fijación ubicado en el centro del monitor y debía responder tan rápido como podía, señalando la dirección donde acababa de ver el cuadrado. Para los efectos de registrar las respuestas direccionales del participante, se configuraron 4 teclas del teclado numérico del PC: La tecla 8 para cuando el cuadrado aparecía arriba ("!); la tecla 5 para cuando aparecía abajo ("!); la tecla 6 , para cuando aparecía a la derecha ('!); y la tecla 4, para cuando aparecía a la izquierda $(\bullet !)$. Estas teclas fueron rotuladas de manera inequívoca, mediante la colocación de etiquetas adhesivas con las flechas respectivas.

En esta tarea, se esperaba que los jugadores intensivos se beneficiaran mucho más que los jugadores moderados y los no jugadores en los ensayos en que hubiese congruencia entre la dirección hacia la que apuntaba el estímulo inductor subliminal (prime) y la localización de la aparición subsecuente del cuadrado.

\section{Procedimiento}

La administración de esta tarea se efectuó bajo las mismas condiciones que la tarea descrita en el experimento anterior. Para neutralizar los posibles efectos de aburrimiento o fatiga, se contrabalanceó el orden de administración de ambas tareas.

\section{Resultados}

El desempeño de los tres grupos de participantes en la tarea de Inducción de Respuestas Direccionales fue comparado, primero, en relación al número de aciertos, vale decir, al porcentaje de veces en que la tecla apretada por el sujeto coincidía con la dirección donde se había presentado el cuadrado. En esta medida todos los grupos mostraron niveles de acierto muy similares con medias de 90 , 93 y 87 y desviaciones estándar de 3, 2,6, y 3,4 por ciento, para los grupos Intensivo, Moderado y NoJugadores, respectivamente (ver Tabla 2).

Tabla 2

Medias y desviaciones estándar de respuestas acertadas de los participantes en los grupos de jugadores intensivos, jugadores moderados y nojugadores, en la tarea de Inducción Direccional de Respuesta

\begin{tabular}{lccc}
\hline Grupo & $\boldsymbol{n}$ & Media & $\begin{array}{c}\text { Desviación } \\
\text { Estándar }\end{array}$ \\
\hline Intensivo & 18 & 90 & 3 \\
Moderado & 15 & 93 & 2,6 \\
No-jugador & 15 & 87 & 3,4 \\
\hline
\end{tabular}

Los datos correspondientes a los tiempos de reacción de los participantes de cada grupo frente a los ensayos con congruencia prime y sin congruencia prime se presentan en el gráfico de la Figura 2.

Con estos datos se procedió a calcular una análisis de varianza de dos vías, con tiempo de reacción como factor intrasujetos, y experiencia con video juegos y "congruencia prime", como factores ínter sujetos. Este análisis mostró la existencia de un efecto principal de grupos, o "experiencia con videojuegos", no significativo, con un $F_{(2,45)}=3,086$, y $p=0,055$; y un efecto principal de "congruencia" con un coeficiente $F_{(1,45)}=4,66, p<0,05$. El efecto interactivo entre estos factores no alcanzó niveles de significación estadística $F_{(2,45)}=0,32$, n.s. En consecuencia, tres grupos de participantes tuvieron tiempos de reacción significativamente menores en los ensayos con congruencia prime $(p<0.05)$; sin embargo, no se encontraron diferencias significativas entre los grupos.

Los resultados de este experimento, si bien se insinúan en la dirección prevista (hipotetizada), ellos no permiten concluir de manera inequívoca que el desempeño de los jugadores intensivos se vea significativamente favorecido por el inductor subliminal 


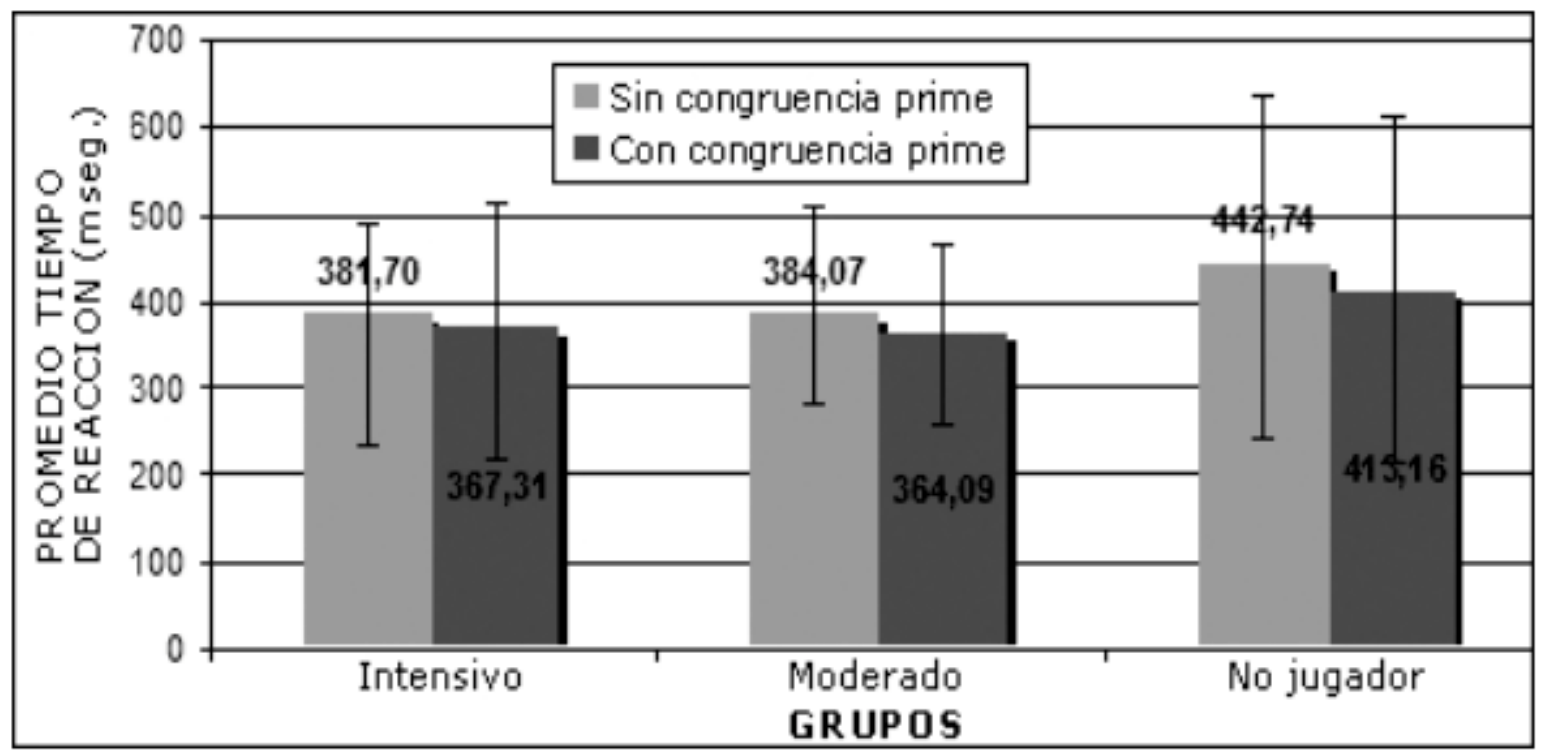

Figura 2. Promedios y desviaciones estándar de los tiempos de reacción de las respuestas en los grupos de jugadores intensivos, moderados y no-jugadores en los ensayos con congruencia prime y sin congruencia prime de la tarea de Inducción Direccional de Respuesta.

de anticipación direccional en los ensayos congruentes, comparado con el desempeño de los participantes de los grupos de jugadores moderados y no-jugadores. De hecho, los resultados de esta tarea muestran que los sujetos en todos los grupos pudieron responder con ventaja en los ensayos congruentes en contraste con los incongruentes; y que si bien ambos grupos de jugadores exhiben un mejor aprovechamiento de los ensayos congruentes que los no jugadores, la diferencia en los tiempos de reacción de sus respuestas en relación con los no jugadores, no alcanzan niveles de significación estadística.

\section{Discusión}

Los principales resultados de este estudio pueden resumirse como sigue: (1) A juzgar por las altas y similares tasas de respuestas correctas logradas por los tres grupos de participantes en las dos tareas escogidas, éstas (las tareas) fueron eficaces en mantener la atención de los participantes y ofrecer así un sustrato estable y comparable contra el cual comparar las posibles diferencias que pudieran ocurrir en los 'tiempo de reacción', producto de los diferentes niveles de experiencia con videojuegos. (2)
La variable 'tiempo de reacción', respecto de la cual se hipotetizó que operaría un efecto de facilitación (reduciendo el tiempo de reacción) en el caso de los jugadores intensivos de videojuegos, permitió confirmar sólo parcialmente tal predicción, por cuanto sólo en la tarea TRS, y dentro de ella, únicamente en comparación con los jugadores moderados, se observó la reducción de tiempo pronosticada. (3) La interpretación de los resultados obtenidos con la tarea de IDR es poco clara. Por una parte, podría decirse que el estimulo inductor subliminal (flecha) fue eficaz para facilitar las respuestas en los ensayos con congruencia prime por parte de todos los participantes. Pero ese mismo patrón de resultados pudo haberse obtenido si la flecha produjo confusión y retardo en las respuestas en los ensayos sin congruencia prime. La tarea no fue construida con los resguardos necesarios para saber cual de los dos efectos está operando, o si ambos lo están. Lo único que podemos afirmar con certeza es que la tarea tal cual fue diseñada, sea por la vía de la facilitación en los ensayos congruentes o por interferencia en los ensayos incongruentes, afectó por igual a todos los sujetos, independientemente de su grado de experiencia previa con videojuegos. 
Cabe señalar que en el presente estudio se optó por explorar el posible efecto facilitador de la experiencia con $\mathrm{VJ}$ con mediciones muy preliminares, tipo sondeo; en otras palabras, se utilizaron muy pocos ensayos para captar el efecto. El objetivo fue medir algo que se suponía estaba presente en los participantes, precisamente, producto de su experiencia con videojuegos, y no algo que tuviera que generarse como consecuencia de un largo entrenamiento. En consecuencia, un enfoque diferente pudo haber sido el de administrar decenas y hasta centenares de bloques de ensayos y comprobar si la experiencia con videojuegos permite obtener mejor aprovecho del entrenamiento. No existe evidencia en nuestro conocimiento que nos permita anticipar cual de estos dos enfoques, u otro diferente, pudiera ser más efectivo para hacer emerger las ventajas que, a juzgar por los resultados del presente estudio, parecieran existir a favor de los videojugadores cuando se trata de aprehender las regularidades implícitas en el ambiente, especialmente en ambientes mediados por tecnología (Rehbein, Alonqueo, Perez-Luco \& Labbé, no prelo).

De lo comentado anteriormente se desprende que una de las razones por la cual los resultados encontrados fueron poco robustos y no concluyentes es la inadecuación de algún aspecto o la totalidad de las tareas experimentales utilizadas. En otras palabras, aparte de revisar y mejorar éstas, convendría buscar también otros dispositivos experimentales que permitan continuar estudiando la relación entre videojuegos y aprendizaje implícito.

Un ejemplo interesante y pionero, en el que adopta un enfoque más complejo y más claramente transferible (o extrapolable) a otras áreas de desempeño cognitivo de los individuos, es el adoptado por Rosas y colaboradores (Rosas y cols., 2000; Thibaut \& Rosas, 2007). Ellos, a pesar de la dificultad que tiene implementar el paradigma de gramáticas artificiales han tenido éxito en adaptar dicho paradigma experimental y han logrado demostrar aprendizaje implícito en diferentes grupos especiales de población escolar (Thibaut \& Rosas, 2007). Estos avances permitirán adoptar o diseñar tareas de mayor complejidad que permitan continuar explorando la relación entre videojuegos y aprendizaje implícito.
Por otra parte, mientras el estudio del aprendizaje implícito en (y a causa de) los videojuegos se perfila como campo de estudio todavía incipiente y promisorio, parece necesario tener presente que ninguno de los dos términos de la ecuación que buscamos resolver, está a su vez completamente caracterizado y satisfactoriamente definido. Por un lado, estamos ciertos que la existencia del aprendizaje implícito ha sido empíricamente demostrada más allá de toda duda (ver Stadler \& Frensch, 1998; Cleeremans, 1997; Sun, 1998, para revisiones de la evidencia empírica reunida). Sin embargo, este cuerpo de evidencia esta dividido en torno a problemas centrales, tales como si el aprendizaje implícito implica la posibilidad de una cognición inconsciente, o si el conocimiento adquirido de manera implícita posee propiedades similares a las que caracterizan al conocimiento adquirido explícitamente (por ejemplo, si puede tener carácter potencialmente abstracto, o si es transferible y generalizable, etc.).

Por otro lado, los videojuegos configuran uno de los conglomerados más diversos y disímiles de productos generados con base en tecnología digital, y orientado al ávido consumo recreativo de niños y jóvenes. Los juegos difieren en aspectos tan diversos como, su tipo, temática, grado de interactividad, habilidades y destrezas necesarias para su uso (Estalló, 1997; Gros, 2000; Gee, 2003). Vistas así las cosas, cuesta imaginar qué tipo experiencia con qué tipo de videojuego podría redundar en qué tipo de aprendizaje implícito. En consecuencia, si se quiere avanzar en esta línea de investigación será necesario tipificar de manera más precisa la naturaleza de las demandas cognitivas que se asocian a cada tipo de videojuego; y al mismo tiempo, diseñar y perfeccionar las tareas y procedimientos experimentales ajustados para registrar los efectos que buscamos detectar y medir.

\section{Referencias}

Alonqueo, P., \& Rehbein, L. (no prelo). Usuarios habituales de videojuegos: Un estudio inicial. Última Década.

Alfagame, B., \& Sánchez, P. (2003). Un instrumento para evaluar el uso y actitudes hacia los videojuegos. Píxel, Bit, Revista de Medios y Educación, 20, 17-32. 
Barlow, H. (2001). The exploitation of regularities in the environment by the brain. Behavioral and Brain Sciences, 24, 602-607.

Cleeremans, A. (1997). Principles for implicit learning. In D. Berry (Ed.), How implicit is implicit learning? (pp. 196-234). Oxford: Oxford University Press.

Cleeremans, A., \& McClelland, J. L. (1991). Learning the structure of event sequences. Journal of Experimental Psychology: General, 120, 235-253.

Cohen, A., Ivry, R., \& Keele, S. W. (1990). Attention and structure in sequence learning. Journal of Experimental Psychology: Learning, Memory, and Cognition, 16, 17-30.

Diez, J., Terrón, E., García, M., Rojo, J., Rufino, C., \& Blanco, D. (2004). La diferencia sexual en el análisis de los videojuegos. Madrid: Instituto de la Mujer y Ministerio de Educación y Ciencia.

Estalló, J. (1997). Videojuegos, efectos sobre el comportamiento. Psicothema, 6, 181-190.

Estalló, J. (2000). Videojuegos: Efectos psicológicos. Revista de Psiquiatría Infantil y Juvenil, 8, 106-116.

Gee, J. P. (2003). What Videogames have to teach us about learning and literacy. New York: Palmgrave MacMillan.

Gros, B. (2000). El ordenador invisible: Hacia la apropiación del ordenador en la enseñanza. Barcelona: Gedisa.

Gros, B., Aguayo, J., \& Almazán, L. (1998). Creación de materiales para la innovación educativa con nuevas tecnologías. Málaga: Imagraf.

Jackson, G. M., \& Jackson, S. R. (1995). Do measures of explicit learning actually measure what is being learned in the serial reaction time task? Psyche, 2(20). Recuperado em 22 fevereiro 2008, de http://psyche.cs.monash.edu.au/v2/ psyche-2-20-jackson.html

Jiménez, L., \& Méndez, C. (1999).Which attention is needed for implicit sequence learning. Journal of Experimental Psychology: Learning, Memory, and Cognition, 25, 236-259.
Jiménez, L., \& Méndez, C. (2001). Implicit sequence learning with competing explicit cues. Quarterly Journal of Experimental Psychology, 54A, 345-369.

Jiménez, L., Vaquero, J. M. M., \& Lupiáñez, J. (2006). Qualitative differences between implicit and explicit sequence learning. Journal of experimental psychology: Learning, Memory, and Cognition, 32, 475-490.

Nissen, M. J., \& Bullemer, P. (1987) Attentional requirements of learning: Evidence from performance measures. Cognitive Psychology, 19, 1-32.

Okagaki, L., \& Frensch, P. (1994). Effects of video game playing on measures of spatial performance: Gender effects in late adolescence. Journal of Applied Developmental Psychology, 15, 33-58.

Reber, A. S. (1967). Implicit learning of artificial grammars. Journal of Verbal Learning and Verbal Behavior, 6, 855-863.

Reber, A. S (1993). Implicit learning and tacit knowledge. Oxford: Oxford University Press.

Rehbein, L., Alonqueo, P., Perez-Luco, R., \& Labbé, C. (no prelo). Callos cognitivos: Nuevos desafíos de la tecnología para la Psicología. Universum (Talca).

Rosas, R., Nussbaum, M., Grau, V., López, X., Salinas, M., Flores e cols. (2000). Más allá del Mortal Kombat: Diseño y evaluación de videojuegos educativos para lenguaje y matemáticas del nivel básico 1. Psykhe, 9(2), 125-141.

Shepard, R. N. (1994). Perceptual-cognitive universals as reflections of the world. Psychonomic Bulletin \& Review, 1, 2-28.

Stadler, M. A., \& Frensch, P. A. (1998). Handbook of implicit learning. Sage: Thousand Oaks.

Thibaut, C., \& Rosas, R. (2007). Diseño de juegos basados en el paradigma de gramáticas artificiales para favorecer el aprendizaje implícito en niños. Psykhe, 16(2), 55-68.

Sun, Y. (1998). Aprendizaje Implícito: Aspectos críticos de su definición y algunas de sus implicancias. Psykhe, 7(2), 41-51. 
Artículo recibido en 09/03/2007.

Aceptado para publicación 15/04/2008.

Este estudio fue financiado por FONDECYT a través del proyecto $N^{\circ} 1040282$ “Aprendizaje implícito en usuarios intensivos de tecnología hipermedial", adjudicado a los dos primeros autores. Resultados preliminares del estudio fueron presentados en la $113^{\text {a }}$ Convención Anual de la American Psychological Association (APA), que tuvo lugar entre el 18 y el 21 de Agosto de 2005, en Washington, D.C., U.S.A.

Dirección postal:

Prof. Dr. Lucio Rehbein Felmer. Universidad de La Frontera. Av Francisco Salazar 01145. Temuco, CP 64811230. Chile.E-mail: brehbein@ufro.cl

Lucio Rehbein Felmer es Profesor Titular de la Facultad de Educación y Humanidades de la Universidad de La Frontera, Chile.

Paula Alonqueo Boudon es Profesor Asistente de la Facultad de Educación y Humanidades de la Universidad de La Frontera, Chile.

Michael Filsecker es licenciado en Psicología e integrante de la Unidad de Estudios y Evaluación del Instituto de Informática Educativa de la Universidad de la Frontera. 